\title{
Soldadura de aceros Dual Phase en chapa fina: GMAW, PAW y RSW
}

\author{
(Welding of Dual Phase steel sheet: GMAW, PAW and RSW)
}

\author{
Hernán Svoboda ${ }^{1,2,3}$; Hernán N. Lorusso, ${ }^{1,4}$; Alejandro Burgueño, 4 \\ ${ }^{1}$ Universidad de Buenos Aires, Facultad de Ingeniería, Departamento de Ingeniería Mecánica, Ciudad Autónoma de Buenos \\ Aires, Argentina. hsvobod@fi.uba.ar \\ ${ }^{2}$ Universidad de Buenos Aires, Facultad de Ingeniería, INTECIN, Laboratorio de Materiales y Estructuras, Ciudad Autónoma de \\ Buenos Aires, Argentina. \\ ${ }^{3}$ Consejo Nacional de Investigaciones Científicas y Técnicas, Ciudad Autónoma de Buenos Aires, Argentina. \\ ${ }^{4}$ Instituto Nacional de Tecnología Industrial, Centro de Investigación y Desarrollo en Mecánica, San Martín, Buenos Aires, \\ Argentina.hlorusso@inti.gob.ar, burgueno@inti.gob.ar
}

\begin{abstract}
Resumen
Los aceros Dual Phase (DP) han encontrado recientemente una fuerte aplicación en elementos estructurales en la industria automotriz, debido a la necesidad de disminuir peso. La soldadura de estos materiales cobra particular importancia considerando su aplicación estructural y los procesos relacionados en su fabricación. En particular la soldadura de resistencia por punto (RSW) y semiautomática con alambre macizo y protección gaseosa (GMAW) son ampliamente utilizados en la industria automotriz. El proceso de soldadura por plasma (PAW) se caracteriza, entre los procesos de soldadura por arco, por ser el de mayor densidad de energía, presentando particular interés en aplicaciones de la industria automotriz (tailor welded blanks). El objetivo del presente trabajo fue estudiar la evolución microestructural y las propiedades de aceros DP soldados mediante los procesos RSW, GMAW y PAW. A este fin, se soldaron cuatro grados de aceros DP con resistencias mecánicas de 550, 700 y 850 MPa en espesores de 1 y 1,3 mm mediante los mencionados procesos. Se caracterizaron las microestructuras y se determinaron las propiedades mecánicas de las uniones soldadas para cada caso. Para los tres procesos se obtuvieron uniones soldadas de calidad satisfactoria. Se observó para todas las soldaduras, que en la ZAC se produce una disminución de la dureza por debajo del valor del material base, relacionada a la descomposición de la fase martensítica. Las soladuras por arco fueron las más afectadas.
\end{abstract}

Palabras clave: aceros Dual Phase, PAW, GMAW, RSW, ZAC

Abstract: Dual Phase steels (DP) have been used recently as an interesting option for structural elements, specialy in automotive industry, due to weight reduce requirements. Welding of these materials becomes particularly important considering their application as structural elements and the related manufacturing methods. In particular resistance spot welding (RSW) and gas metal arc welding $(G M A W)$ are widely used in the automotive manufacturing. The plasma arc welding (PAW) has the charateristic, within arc welding processes, to involve the highest energy density, being this parameter interesting to certain applications on automative industry (tailor welded blanks). The objective of this work is to study the microstructural evolution and properties of welded DP steels by mean of RSW, GMAW and PAW. In this regard four DP steels, with tensile strength of 550, 700 y 850 MPa and thickness of 1 and 1.3 mm, were welded by mean of mentioned welding processes. Microstructures were charaterized and mechanical properties for each weld joint were determined. For the three processes good quality welded joints were obtained. It was observed on heat affected zone (HAZ) a softened zone whose hardness is below to initial base metal hardness. This softening is related to the dissolution of the martensitic phase due to thermal cycle introduced by welding. Arc welds were the most affected.

Key-words: welding, Dual Phase steel, PAW, GMAW, RSW, HAZ softening

\section{Introducción}

En los últimos años han tenido lugar numerosos desarrollos relacionados con la tecnología y fabricación de nuevos aceros

(Recebido em 16/05/2011; Texto final em 30/06/2011).

Artigo originalmente publicado no CONSOLDA 2010 vinculados con la disminución de peso y aumento de la seguridad de vehículos. En este sentido los Aceros Avanzados de Alta Resistencia (AHSS) han capturado la mayor atención $[1,2]$. Sin embargo, junto con las ventajas que proveen estos nuevos aceros surge la necesidad de generar conocimiento sobre su conformabilidad y soldabilidad [1, 2]. Dentro de los AHSS se encuentran los aceros denominados Dual Phase (DP) de alta resistencia que presentan una mayor conformabillidad para diseños que involucren partes más complejas. Dichos 
aceros, consisten en una matriz ferrítica que contiene una fracción variable de fase martensítica de alta dureza. La fracción de segunda fase martensítica, aumenta con el aumento de la resistencia deseada del acero. La matriz ferrítica blanda es generalmente continua, proveyendo una excelente ductilidad. Cuando estos aceros se conforman, la deformación se concentra en la fase ferrítica blanda, rodeando las "islas" de martensita, generando un alto coeficiente de endurecimiento por deformación para estos materiales $[1,3,4]$. Esto, sumando a un excelente alargamiento a rotura, provee a estos aceros de una mayor resistencia a la tracción que los aceros convencionales con similar tensión de fluencia. En general, el contenido de carbono se encuentra alrededor de $0,10-0,15 \%$, que junto a la presencia de Mn (1-2\%), Si (0,03-0,4\%) Cr, Mo y Ni ( 0,2\%) facilitan la templabilidad [1], permitiendo la formación de martensita a velocidades de enfriamiento tecnológicamente aceptables. A su vez, el $\mathrm{C}$ ejerce también un efecto endurecedor de la martensita. El tamaño de grano de la matriz ferrítica es otro de los parámetros que controlan el endurecimiento y la buena tenacidad de estos materiales [3], por ello la presencia de microaleantes $\mathrm{V}, \mathrm{Nb}$, Ti $(\sim 0,030 \%)$ es favorable a las propiedades finales de los aceros DP. Estos aceros de última generación, se aplican en partes estructurales, especialmente en la industria automotriz, presentando una alta resistencia mecánica del orden de 500 a $1000 \mathrm{MPa}$, manteniendo muy buena conformabilidad. Sin embargo, existen diversos materiales base (aceros microaleados, aceros de grano ultrafino, aceros al C-Mn, etc.) a partir de los cuales, pueden obtenerse dichos aceros DP, siendo escasos los estudios sistemáticos realizados al respecto [1]. Los aceros DP en los últimos años han incrementado su utilización en partes estructurales de automóviles, las cuales se encuentran soldadas en muchas de sus aplicaciones [1,2,5-7].

Por otro lado, la soldabilidad de estos materiales es un aspecto clave en cuanto a sus aplicaciones, por lo que el estudio de la evolución microestructural y de las propiedades de la unión soldada, es un tópico de gran interés, especialmente en relación a los procesos de soldadura por arco eléctrico con protección gaseosa y alambre macizo (GMAW) y soldadura por resistencia de punto (RSW), de gran aplicación en la industria automotriz. El proceso de soldadura por plasma (PAW) se caracteriza, entre los procesos de soldadura por arco, por ser el de mayor densidad de energía [5]. En los procesos de soldadura, este aspecto presenta una fuerte influencia sobre la relación de aspecto del cordón, el flujo de calor, etc. En este sentido, el ciclo térmico introducido por la soldadura, modifica la estructura ferrítico-martensítica original, generando variaciones de las propiedades en la Zona Afectada por el Calor (ZAC). Estas variaciones se encuentran asociadas al revenido de la martensita, lo que produce en la zona del material recalentada hasta unos $700^{\circ} \mathrm{C}$ (Zona Sub-Crítica) la formación de una estructura ferrítica con carburos, de baja dureza [6-8]. Las propiedades mecánicas resultantes de la junta se verán asociadas a las características de dicha zona. Las características diferenciales de cada uno de estos procesos en relación al ciclo térmico que introducen podrán presentar efectos diferentes sobre la evolución microestructural del material. Asimismo, los espesores involucrados en este tipo de aplicaciones son, en general, delgados $(<2,5 \mathrm{~mm})$, lo que requiere un procedimiento de soldadura adecuadamente especificado. La información disponible respecto de estudios sistemáticos realizados en este sentido es escasa. El objetivo del presente trabajo es estudiar la soldadura de distintos grados de aceros DP, obtenidos a partir de aceros comerciales, mediante los procesos GMAW, PAW y RSW, debido a que los mismos son empleados en la industria automotriz. Se analizará la evolución microestructural y de las propiedades mecánicas de las uniones soldadas, buscando una mejor comprensión de los fenómenos metalúrgicos que tienen lugar debido al ciclo térmico de la soldadura y su efecto sobre las propiedades.

\section{Materiales y Métodos}

Se soldaron cuatro tipos de aceros DP con los mencionados procesos de soldadura. En la Tabla 1, se muestran los materiales analizados y sus características.

Estos grados de materiales se seleccionaron debido a que se encuentran dentro del rango de espesores y resistencias de interés en las aplicaciones de la industria automotriz [1].

Para la obtención de los aceros DP analizados, se partió de chapas base de un acero convencional C-Mn y tres microaleados. Se determinó, para cada chapa, la composición química mediante espectrometría de emisión óptica (OES) y se realizaron tratamientos térmicos sobre cada material, a fin de obtener los niveles de resistencia deseados.

Estos parámetros, se definieron a partir de los resultados obtenidos en trabajos previamente publicados por los autores [8]. Luego de obtenidos los grados de DP deseados, se realizó la caracterización microestructural, mediante microscopía óptica (LM). La cuantificación de fases se realizó de acuerdo con ASTM E 562 [9]. Se determinó la microdureza Vickers (HV 1) de acuerdo a la norma ASTM E384 [10], reportando los valores promedio de cinco mediciones junto con su desvío estándar. Se obtuvieron las propiedades en tracción, según ASTM E 8 [11], y se realizaron ensayos de embutido tipo Olsen, utilizando como guía la norma ASTM E 643 [12].

Posteriormente, con los diferentes grados de DP, se soldaron a tope probetas de $70 \mathrm{~mm}$ de ancho por $150 \mathrm{~mm}$ de longitud, mediante el proceso de soldadura semiautomática con alambre macizo y protección gaseosa (GMAW) y por plasma (PAW), en ambos casos el proceso fue mecanizado. Para GMAW el alambre empleado fue AWS A5.18 ER70S-6 de 0,8 mm de diámetro, en transferencia cortocircuito, y se utilizó Ar-18\% CO $\mathrm{CO}_{2}$ como gas de protección, con un caudal de $151 . \mathrm{min}^{-1}$. En el caso de PAW la soldadura fue autógena y se utilizó argón como gas de plasma y de protección, con caudales de 0,4 y $131 . \mathrm{min}^{-1}$, respectivamente. En ambos casos se empleó un respaldo de aluminio de $5 \mathrm{~mm}$ de espesor. Para el proceso GMAW la distancia pico de contactopieza fue de $9 \mathrm{~mm}$. La distancia pieza-tobera para la soldadura por plasma fue de $6 \mathrm{~mm}$. Los parámetros eléctricos y las velocidades de soldadura se muestran en la Tabla 2. A partir de las muestras soldadas, se realizó la caracterización macro y microestructural, se determinó el perfil de microdureza, así como las propiedades en tracción y embutido tipo Olsen, evaluándose las variaciones de microestructura y de propiedades, luego de la soldadura. 
Tabla 1. Aceros DP analizados.

\begin{tabular}{|l|l|c|c|}
\hline \multicolumn{1}{|c|}{ Material } & \multicolumn{1}{c|}{ Tipo } & Espesor $[\mathrm{mm}]$ & Resistencia a la tracción [MPa] \\
\hline $\mathrm{C}-\mathrm{Mn}$ & carbono-manganeso & 1,0 & 550 \\
\hline $\mathrm{Nb}$ & microaleado al $\mathrm{Nb}$ & 1,0 & 700 \\
\hline $\mathrm{Ti}-\mathrm{Nb}$ & microaleado al Ti-Nb & 1,0 & 850 \\
\hline Ti-Nb-T & microaleado al Ti-Nb & 1,3 & 700 \\
\hline
\end{tabular}

Tabla 2. Parámetros eléctricos y velocidad de soldadura para los procesos por arco.

\begin{tabular}{|c|c|c|c|c|c|}
\hline Material & Proceso & $\begin{array}{c}\text { Corriente de soldadura } \\
{[\mathrm{A}]}\end{array}$ & $\begin{array}{c}\text { Tensión de soldadura } \\
{[\mathrm{V}]}\end{array}$ & $\begin{array}{l}\text { Velocidad de avance } \\
{\left[\mathrm{mm} \cdot \mathrm{min}^{-1}\right]}\end{array}$ & $\begin{array}{c}\text { Calor aportado } \\
{\left[\mathrm{kJ} \cdot \mathrm{mm}^{-1}\right]}\end{array}$ \\
\hline C-Mn & \multirow{4}{*}{ GMAW } & \multirow{3}{*}{50} & \multirow{3}{*}{18} & \multirow{3}{*}{350} & \multirow{3}{*}{0,15} \\
\hline $\mathrm{Nb}$ & & & & & \\
\hline $\mathrm{Ti}-\mathrm{Nb}$ & & & & & \\
\hline Ti-Nb-T & & 80 & 18 & 410 & 0,21 \\
\hline C-Mn & \multirow{4}{*}{ PAW } & \multirow{3}{*}{48} & \multirow{3}{*}{29} & \multirow{3}{*}{390} & \multirow{3}{*}{0,21} \\
\hline $\mathrm{Nb}$ & & & & & \\
\hline $\mathrm{Ti}-\mathrm{Nb}$ & & & & & \\
\hline Ti-Nb-T & & 50 & 29 & 250 & 0,35 \\
\hline
\end{tabular}

Tabla 3. Parámetros de soldadura para el proceso RSW.

\begin{tabular}{|c|c|c|c|c|c|}
\hline Material & Proceso & $\begin{array}{c}\text { Corriente de soldadura } \\
{[\mathrm{kA}]}\end{array}$ & $\begin{array}{l}\text { Fuerza de soldadura } \\
\qquad[\mathrm{kN}]\end{array}$ & $\begin{array}{c}\text { Tiempo de } \\
\text { soldadura [ciclos] }^{1}\end{array}$ & 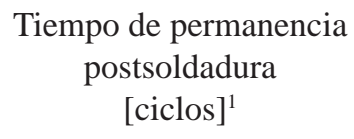 \\
\hline C-Mn & \multirow{4}{*}{ RSW } & \multirow{3}{*}{9} & \multirow{3}{*}{4} & \multirow{3}{*}{6} & \multirow{3}{*}{16} \\
\hline $\mathrm{Nb}$ & & & & & \\
\hline $\mathrm{Ti}-\mathrm{Nb}$ & & & & & \\
\hline Ti-Nb-T & & 10,5 & 4 & 5 & 16 \\
\hline
\end{tabular}

${ }^{1}$ La base de tiempos de ciclos es sobre una frecuencia de $50 \mathrm{~Hz}$.

Tabla 4. Composición química (\% en masa) y carbono equivalente de los aceros analizados.

\begin{tabular}{|c|c|c|c|c|}
\hline & $\mathrm{C}-\mathrm{Mn}$ & $\mathrm{Nb}$ & $\mathrm{Ti}-\mathrm{Nb}$ & $\mathrm{Ti}-\mathrm{Nb}-\mathrm{T}$ \\
\hline $\mathrm{C}$ & 0,060 & 0,090 & 0,088 & 0,070 \\
\hline $\mathrm{Mn}$ & 0,190 & 0,990 & 0,910 & 1,025 \\
\hline $\mathrm{P}$ & 0,008 & 0,018 & 0,024 & 0,009 \\
\hline $\mathrm{S}$ & 0,008 & 0,009 & 0,007 & 0,007 \\
\hline $\mathrm{Si}$ & 0,020 & 0,080 & 0,311 & 0,030 \\
\hline $\mathrm{Ti}$ & 0,001 & 0,001 & 0,075 & 0,018 \\
\hline $\mathrm{Nb}$ & 0,001 & 0,039 & 0,025 & 0,021 \\
\hline $\mathrm{CEn}$ & 0,080 & 0,202 & 0,195 & 0,172 \\
\hline $\mathrm{Pcm}$ & 0,072 & 0,144 & 0,146 & 0,124 \\
\hline
\end{tabular}


Los parámetros de soldadura para el proceso RSW se indican en la Tabla 3. La geometría de las probetas soldadas fue de acuerdo a AWS D8.9 [13]. Los electrodos fueron clase 2, $\mathrm{Cu}-\mathrm{Cr}-\mathrm{Zr}$, de $6 \mathrm{~mm}$ de diámetro con punta truncada a $45^{\circ}$ y refrigerados por agua con un caudal de $101 . \mathrm{min}^{-1} \mathrm{y}$ temperatura $24^{\circ} \mathrm{C}$. Las probetas soldadas fueron evaluadas metalográfica y mecánicamente a través de mediciones de microdureza Vickers, según AWS D8.9 [13] y pruebas de arrancamiento (peel test) de acuerdo a ISO 14270 [14].

\subsection{Aceros DP obtenidos}

En la Tabla 4 se presenta la composición química de los distintos aceros analizados, expresada en porcentaje en peso (wt\%). La chapa identificada como C-Mn presenta un bajo contenido de $\mathrm{C}$, con muy bajos contenidos de $\mathrm{Mn}$ y $\mathrm{Si}$, sin

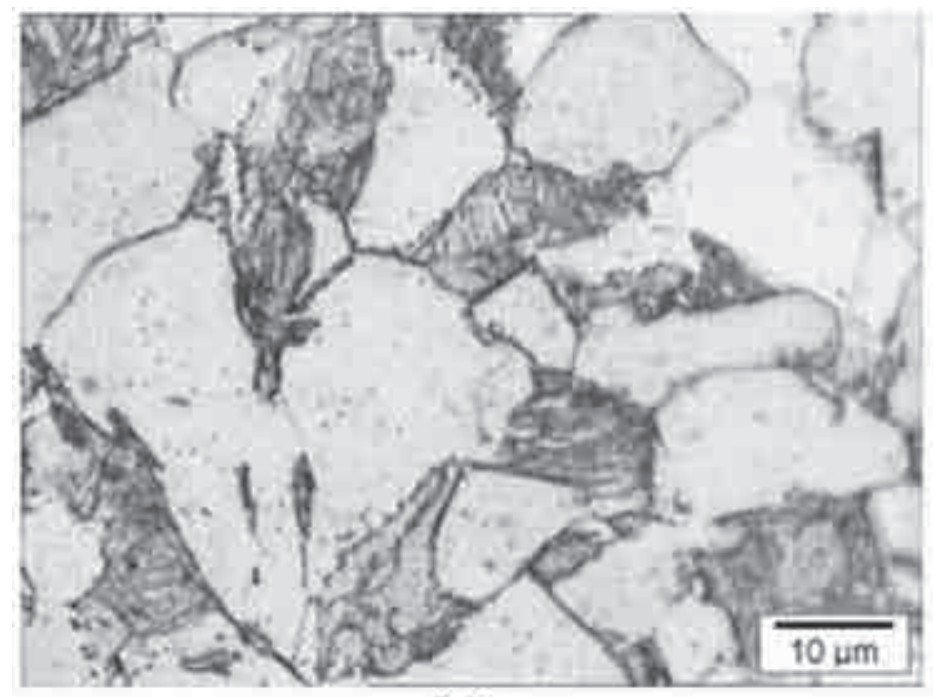

(a)

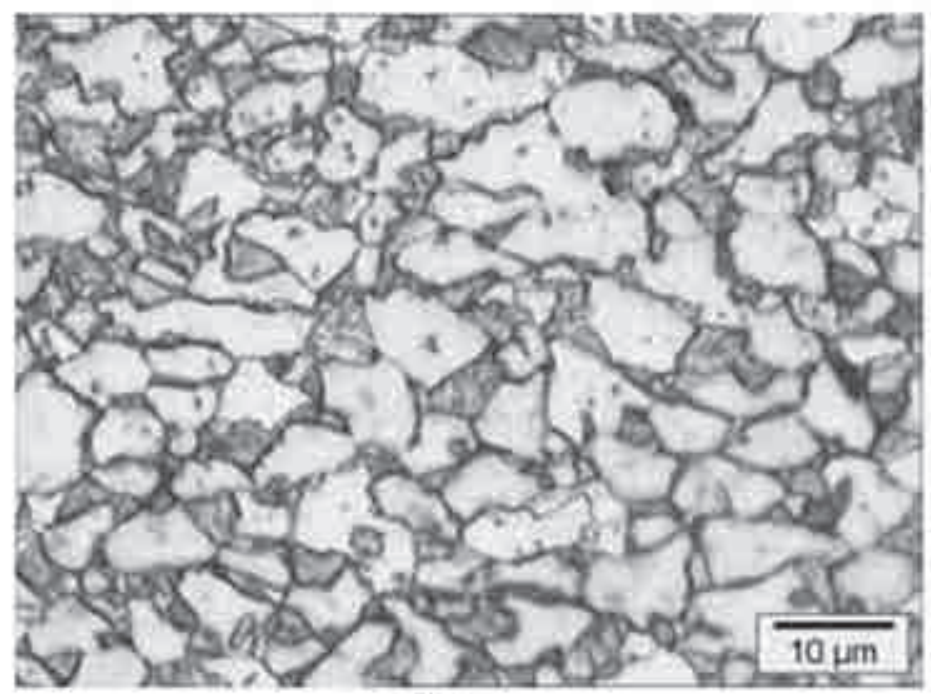

(c) ningún otro contenido de aleantes. La chapa identificada como $\mathrm{Nb}$ presenta un mayor contenido de $\mathrm{C}$ y $\mathrm{Mn}$, con el agregado de $\mathrm{Nb}$ como microaleante. El material identificado como Ti$\mathrm{Nb}-\mathrm{T}$, de mayor espesor, presenta un bajo contenido de $\mathrm{C}$ y $\mathrm{Si}$, similar a la chapa C-Mn, pero con un mayor contenido de $\mathrm{Mn}$, y el agregado de $\mathrm{Ti}$ y $\mathrm{Nb}$ como microaleantes. Finalmente, el material identificado como Ti-Nb, presenta contenidos de $\mathrm{C}$ y $\mathrm{Mn}$ similares a la chapa $\mathrm{Nb}$, pero con mayor contenido de $\mathrm{Si}$, y el agregado Ti como microaleante. Para cada chapa se evaluó el carbono equivalente desarrollado por Yurioka, CEn, y el parámetro de composición del material, Pcm, desarrollado por Ito [15], los cuales aplican a estos aceros por ser de relativo bajo carbono $(<0,1 \%)$. Los máximos valores de CEn y Pcm corresponden a los materiales $\mathrm{Nb}$ y $\mathrm{Ti}-\mathrm{Nb}$.

En la Figura 1, se observan las microestructuras de los aceros DP obtenidos. En todos los casos se trata de microestructuras

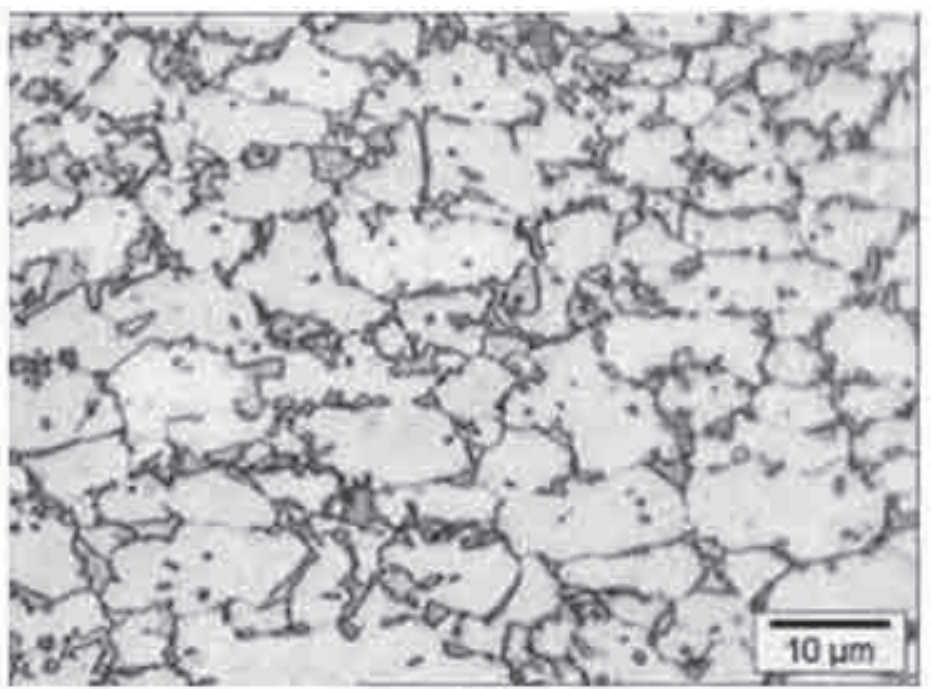

(b)

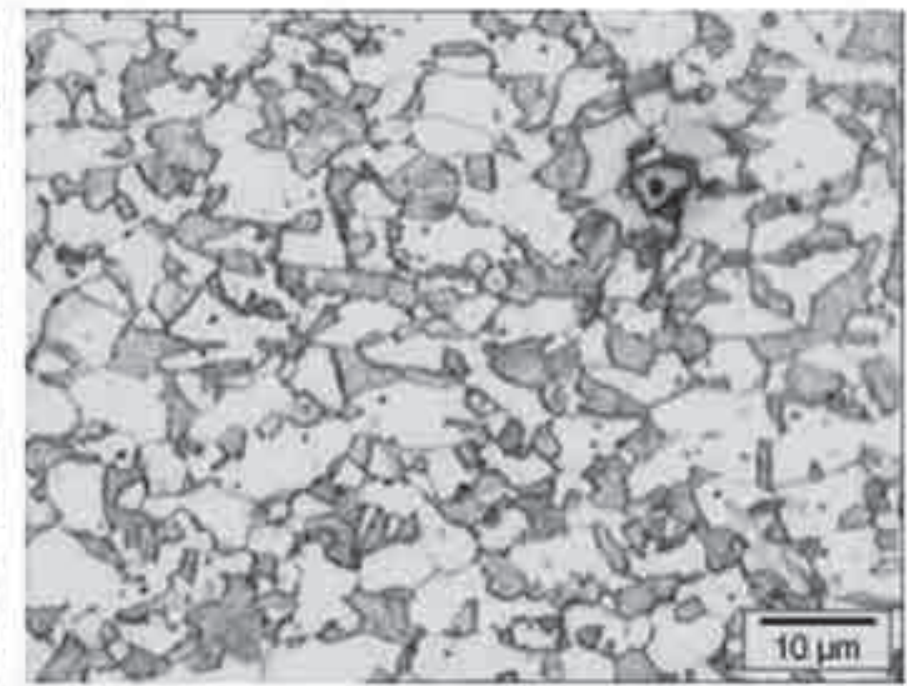

(d)

Figura 1. Microestructuras (LM) de los aceros DP analizados (ataque Nital 2\%): a- C-Mn; b- Nb; c- Ti-Nb-T y d- Ti-Nb. 
ferrítico-martensíticas, con la martensita homogéneamente distribuida y con fracciones correspondientes con la resistencia requerida [8]. Asimismo, los aceros con microaleantes presentan tamaño de grano sensiblemente menor que el acero C-Mn.

En la Tabla 5, se presentan características microestructurales (fracción de martensita, M), propiedades mecánicas (microdureza Vickers, HV1; propiedades en tracción - tensión de fluencia, $\mathrm{R}_{\mathrm{p} 0,2}$; resistencia a la tracción, $\mathrm{R}_{\mathrm{m}}$; alargamiento a la rotura, $\mathrm{A}$ ) y de conformabilidad (altura de copa, $\mathrm{h}_{0}$ ) de los cuatro aceros DP.

Tabla 5. Microdureza, propiedades de tracción y embutido Olsen de los cuatro aceros DP estudiados.

\begin{tabular}{|l|c|c|c|c|c|c|c|}
\hline Material & $\begin{array}{c}\mathrm{M} \\
{[\%]}\end{array}$ & HV 1 & $\begin{array}{c}\mathrm{R}_{\mathrm{p} 0.2} \\
{[\mathrm{MPa}]}\end{array}$ & $\begin{array}{c}\mathrm{R}_{\mathrm{m}} \\
{[\mathrm{MPa}]}\end{array}$ & $\begin{array}{c}\mathrm{A} \\
{[\%]}\end{array}$ & $\begin{array}{c}\mathrm{R}_{\mathrm{m}} / \\
\mathrm{R}_{\mathrm{p} 0,2}\end{array}$ & $\begin{array}{c}\mathrm{h}_{\mathrm{o}} \\
{[\mathrm{mm}]}\end{array}$ \\
\hline C-Mn & 30 & $160 \pm 4$ & 395 & 565 & 16,9 & 1,43 & 7,5 \\
\hline $\mathrm{Nb}$ & 30 & $215 \pm 2$ & 480 & 760 & 11,4 & 1,58 & 10,3 \\
\hline Ti-Nb & 40 & $249 \pm 2$ & 475 & 845 & 14,0 & 1,78 & 9,7 \\
\hline Ti-Nb- T & 40 & $210 \pm 6$ & 410 & 725 & 14,8 & 1,77 & 11,2 \\
\hline
\end{tabular}

En todos los casos se obtuvieron los tres niveles de resistencia buscados $(550,700$ y $850 \mathrm{MPa})$. A su vez, se tiene un alto coeficiente de endurecimiento por deformación plástica para todos los casos, especialmente en las probetas de aceros microealeados, característico de este tipo de materiales [1]. Los valores de alargamiento a la rotura obtenidos se encuentran dentro de lo esperado para este tipo de materiales, reportándose en la literatura que existe un rango de porcentajes de martensita para el cual la ductilidad se ve favorecida [4]. En cuanto al ensayo de embutido, las chapas Ti-Nb, Nb y Ti-Nb-T presentaron alturas de copa, $\mathrm{h}_{0}$, superiores respecto al comportamiento de la chapa C-Mn. Para lo materiales microaleados, los resultados obtenidos son consistentes con el valor de resistencia. El bajo valor obtenido para el acero C-Mn puede estar asociado al mayor tamaño de grano de este material.

\section{Resultados y discusión}

\subsection{Análisis de las soldaduras por arco}

En todos los casos, la soldabilidad fue muy buena sin presentar defectos de soldadura, mostrando un aspecto superficial destacable. En la Figura 2, se observan las macrografías de las soldaduras GMAW y PAW, junto con los perfiles de microdureza realizados sobre las mismas.

En todos los casos se observa la penetración completa. Puede verse que el tamaño de la junta es mayor para la chapa de 1,3 $\mathrm{mm}$ de espesor, asociado a un mayor aporte térmico. Asimismo, el volumen fundido para el proceso PAW es menor que para el GMAW. En los perfiles se observa que la dureza disminuye desde el metal de soldadura hasta alcanzar un valor mínimo que se encuentra en la ZAC para luego aumentar hasta el valor del material base.

Para En la Tabla 6 se muestran los resultados obtenidos de la medición de la longitud promedio de las distintas zonas de las juntas soldadas, medidas sobre la mitad del espesor.

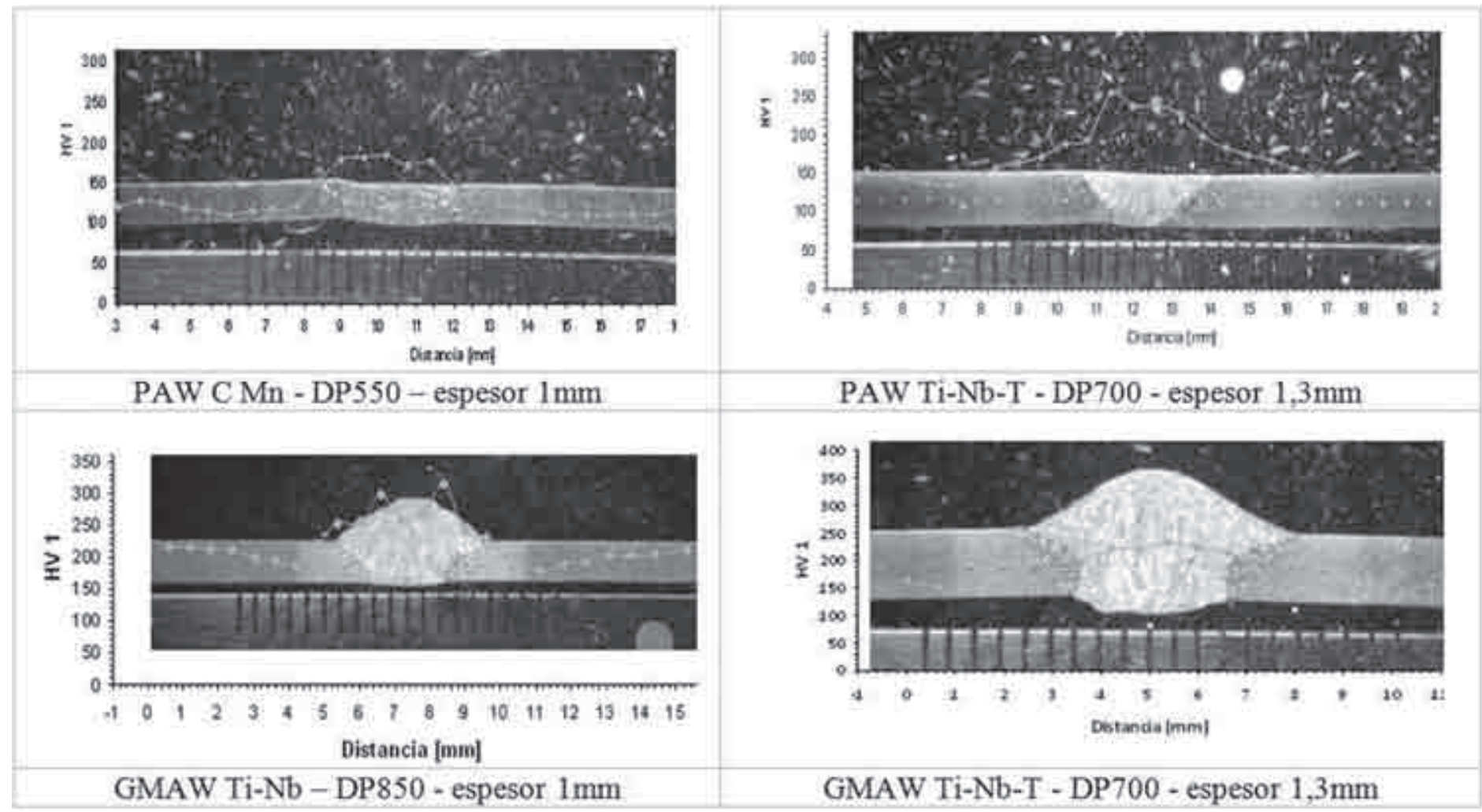

Figura 2. Macrografías perfiles de microdureza HV1 de las uniones soldadas con PAW y GMAW de los aceros DP analizados (distancia entre marcas $0,5 \mathrm{~mm}$ ). 
Tabla 6. Longitud promedio de las diferentes zonas y de la junta soldada para GMAW y PAW.

\begin{tabular}{|c|c|c|c|c|}
\hline \multirow{2}{*}{ Región } & \multicolumn{4}{|c|}{ GMAW - Longitud promedio zonas [mm] } \\
\cline { 2 - 5 } & CMn & Nb & Ti Nb & Ti Nb T \\
\cline { 2 - 5 } & DP 550 & DP 700 & DP 850 & DP 700 \\
\hline MS & 3,5 & 3,4 & 2,9 & 3 \\
\hline ZAC & 9,8 & 10,1 & 9,8 & 11,8 \\
\hline MS+2ZAC & 23,1 & 23,6 & 22,5 & 26,6 \\
\hline
\end{tabular}

\begin{tabular}{|c|c|c|c|c|}
\hline \multirow{2}{*}{ Región } & \multicolumn{4}{|c|}{ PAW - Longitud promedio zonas [mm] } \\
\cline { 2 - 5 } & $\mathrm{CMn}$ & $\mathrm{Nb}$ & $\mathrm{Ti} \mathrm{Nb}$ & $\mathrm{Ti} \mathrm{Nb} \mathrm{T}$ \\
\cline { 2 - 5 } & $\mathrm{DP} 550$ & $\mathrm{DP} 700$ & $\mathrm{DP} 850$ & $\mathrm{DP} 700$ \\
\hline MS & 3,3 & 2,7 & 2,7 & 3 \\
\hline ZAC & 11,5 & 12 & 12 & 12,5 \\
\hline MS+2ZAC & 18,1 & 17,4 & 17,4 & 18,5 \\
\hline
\end{tabular}

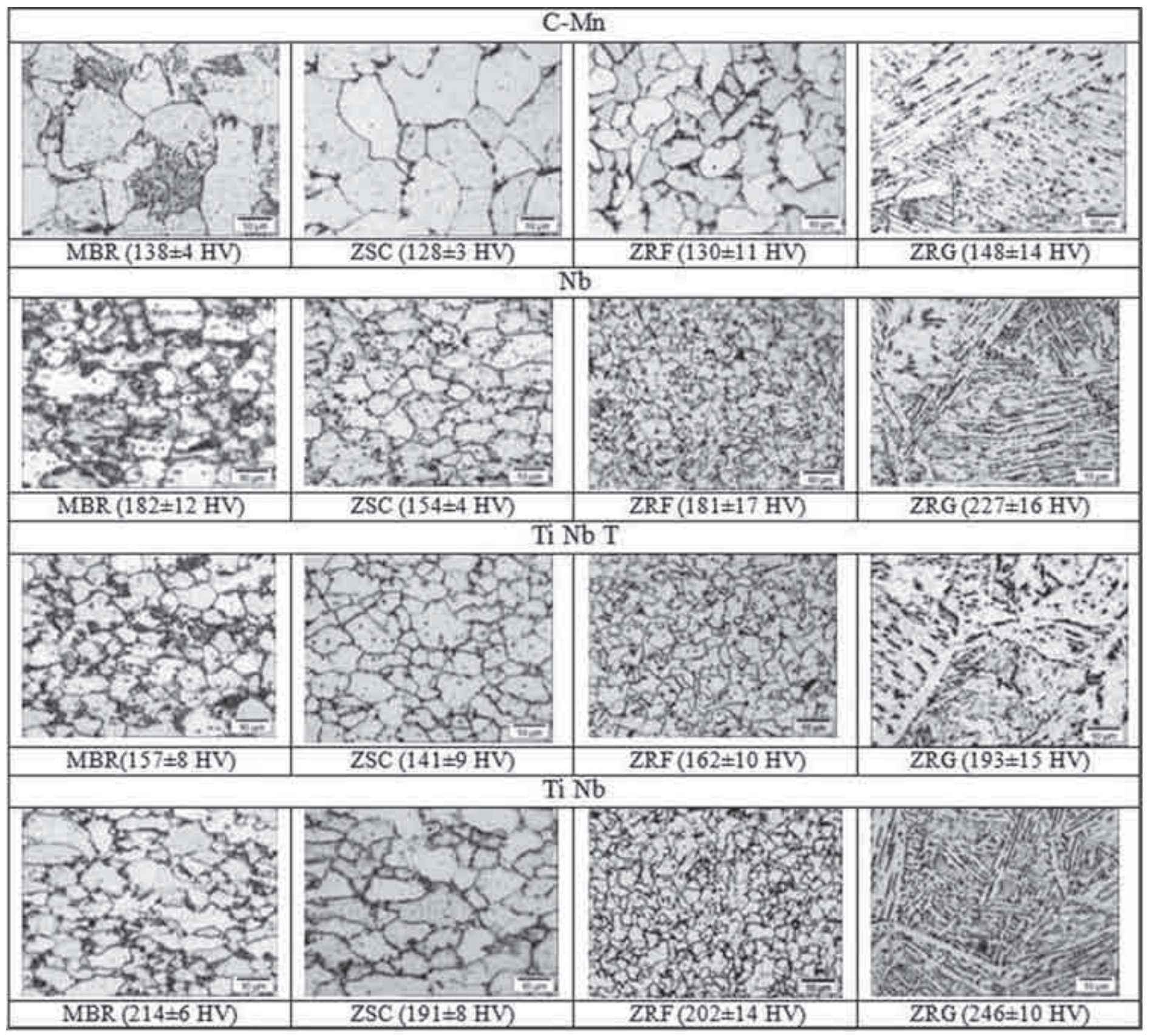

Figura 3. Microestructuras y microdureza de las distintas zonas afectadas térmicamente de las uniones soldadas con GMAW (ataque Nital 2\%): a- C-Mn, b- Nb, c- Ti-Nb-T y d- Ti-Nb. 


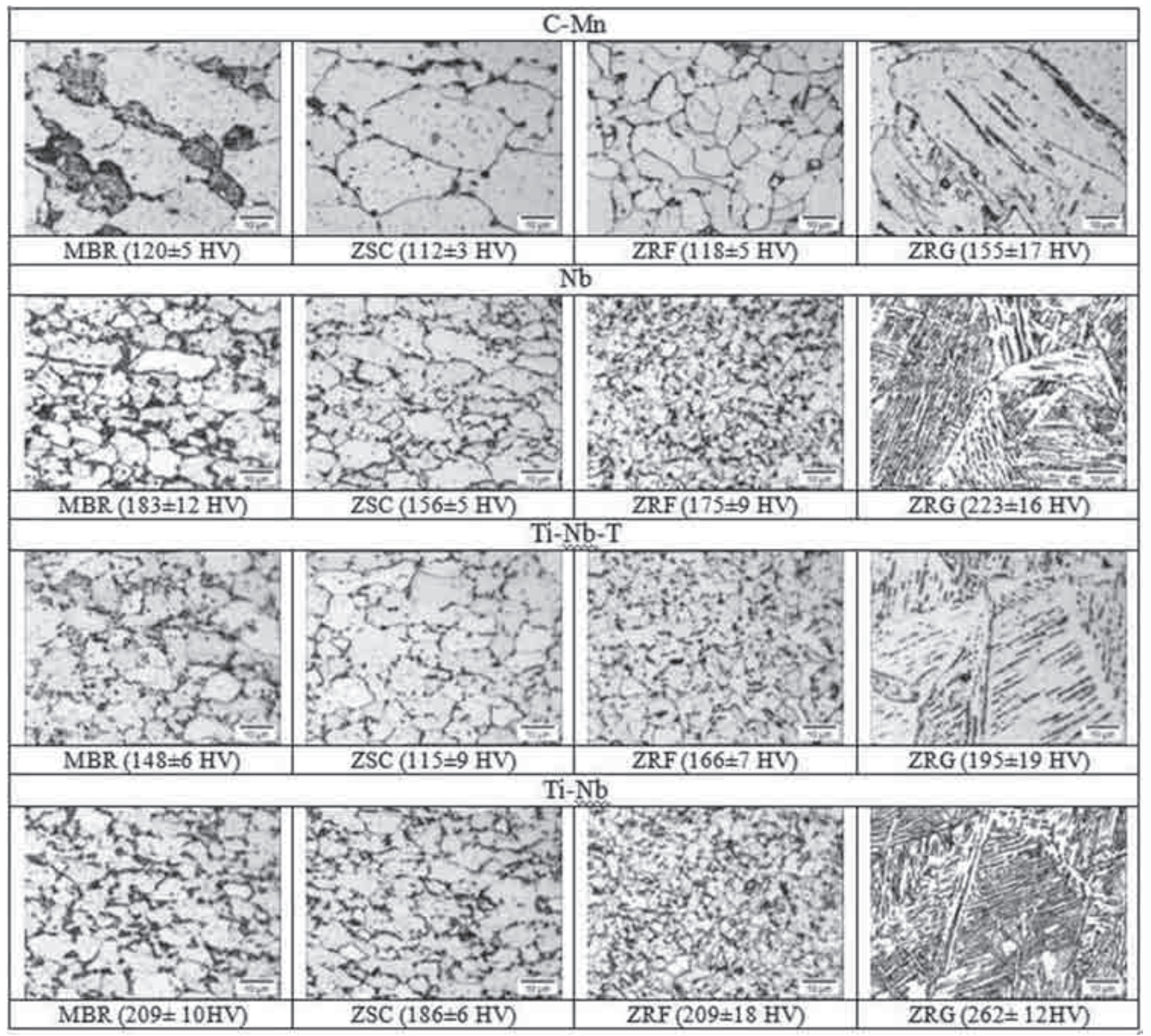

Figura 4. Microestructuras y microdureza de las distintas zonas afectadas térmicamente de las uniones soldadas mediante PAW (ataque Nital 2\%): a- C-Mn, b- Nb, c- Ti-Nb-T y d- Ti-Nb.

Para el caso de las probetas soldadas por GMAW, los tres materiales de $1 \mathrm{~mm}$ de espesor mostraron que el tamaño del MS, la ZAC y la longitud total de la junta son similares, mientras que los resultados obtenidos para la chapa de 1,3 $\mathrm{mm}$ fueron superiores. Esto estaría asociado al mayor aporte térmico introducido en la chapa de mayor espesor. La misma observación puede hacerse para las probetas soldadas por el proceso PAW. A su vez, estas probetas, presentaron longitudes menores que las medidas en las soldadas por GMAW. Es sería debido al aporte térmico adicional asociado a la transferencia metálica del proceso GMAW, a pesar de tener un aporte térmico nominal equivalente al del proceso PAW.
En las Figuras 3 y 4 se muestran detalladamente las microestructuras características que componen las ZAC para las soldaduras GMAW y PAW, respectivamente. Las microdurezas promedio de cada zona también se presentan en tales figuras.

Para todas las juntas, microscópicamente se observaron distintas zonas que se identificaron como MS: metal de soldadura, ZRG: zona recristalizada de grano grueso y ZRF: zona recristalizada de grano fino. Estas zonas son las típicamente observadas en la soldadura por fusión de aceros al carbono y de baja aleación. Además, en mayor o menor medida según el procedimiento de soldadura, se observaron la ZSC: zona subcrítica y el MBR: metal base revenido. La ZSC y el 


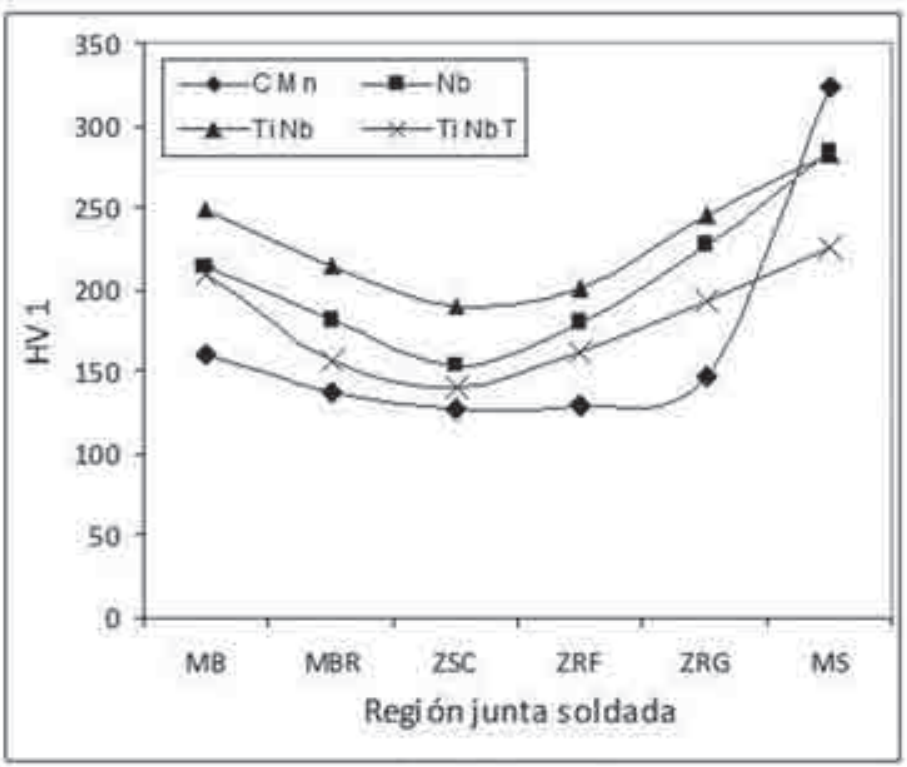

(a) GMAW

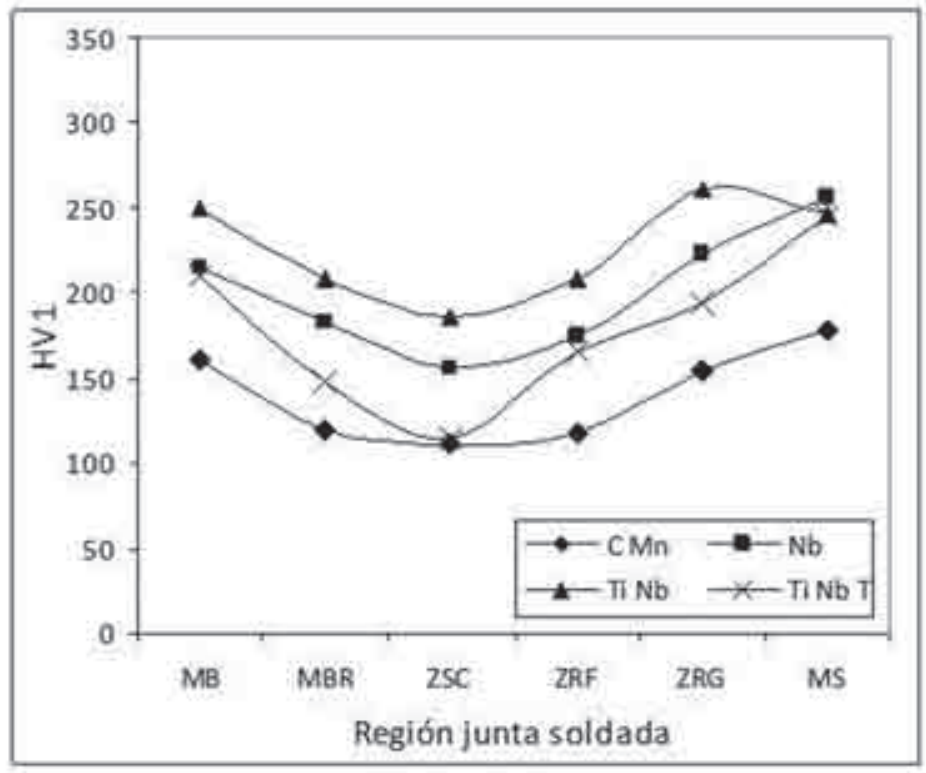

(b) PAW

Figura 5. Microdureza promedio de cada zona de la unión soldada.(a) GMAW; (b) PAW.

MBR corresponden al material que no superó la temperatura $\mathrm{A}_{\mathrm{C} 1}$ pero que debido a que el material base presenta una fracción de martensita, esta fase se reviene durante el calentamiento mencionado y por lo tanto ve modificadas sus propiedades, por lo que forman parte de la ZAC. La diferenciación realizada en el presente trabajo entre ambas zonas radica en que en la ZSC la temperatura alcanzada es levemente inferior a $\mathrm{A}_{\mathrm{Cl}}$ y presenta una microestructura diferente a lo observado en el MBR.

La ZSC fue la que presentó una menor dureza consistentemente con una estructura ferrítica con carburos, sin presencia de martensita, con una tamaño de grano levemente mayor al del material base original. Esto está asociado a que durante el calentamiento de esta zona a una temperatura menor pero cercana a la crítica inferior de transformación, la martensita original se reviene, descomponiéndose a ferrita y carburos. Luego, la dureza final de esta zona estará definida principalmente por el tamaño de grano y el endurecimiento por solución sólida de la ferrita y la distribución de los carburos precipitados. En la ZRF se produce una recristalización por austenización que tiene asociado un afino de grano respecto del tamaño inicial del material base, debido a las relativamente bajas temperaturas alcanzadas, por lo que la microestructura final es fundamentalmente ferrítica de grano equiaxiado fino [16].

Los valores de dureza en esta zona son levemente superiores a los medidos en la ZSC. En la ZRG se tiene un crecimiento de grano significativo debido a las altas temperaturas de austenización alcanzadas lo que produce durante el enfriamiento la descomposición de la austenita en diversas fases ferríticas, principalmente del tipo ferrita con segundas fases alineadas y no alineadas, las que tienen asociada una mayor dureza. Por último, en el MBR se tiene que la martensita original del acero DP se reviene por el ciclo térmico de la soldadura, produciendo un ablandamiento en dicha zona respecto de los valores obtenidos para la condición original, observándose una estructura de martensita revenida y ferrita.

No se observaron diferencias microestructurales significativas entre las probetas soldadas con GMAW y las soldadas por PAW.

En todos los casos se observó que la dureza disminuye desde el MS hacia el metal base, alcanzando el valor mínimo en la transición entre la ZRF y la ZSC, incrementándose luego hasta alcanzar finalmente el valor de la dureza del material base. Esto se representa en la Figura 5 donde se graficó la dureza promedio representativa de cada zona de la junta para ambos procesos.

Puede verse que en general los valores de dureza promedio de las distintas zonas de la ZAC mostraron la misma tendencia al variar los materiales base. El C-Mn presentó los menores valores seguido por el Ti-Nb-T, luego el $\mathrm{Nb}$ y finalmente los mayores valores de dureza se obtuvieron para el $\mathrm{Ti}-\mathrm{Nb}$, consistentemente con los valores de dureza de los materiales base y con los índices CEn y Pcm. Estas tendencias se repitieron tanto para probetas soldadas con GMAW como con PAW, consistentemente con lo observado en la microestructura.

En la Tabla 7, se muestran los resultados obtenidos de los ensayos de tracción y embutido Olsen para las soldaduras producidas con los procesos por arco. También se indica la disminución de resistencia y conformabilidad de las uniones soldadas respecto de los metales base. Tanto la resistencia a la tracción, como la altura de embutido se vieron disminuidas. Una medida de la pérdida de propiedades son los índices $\mathrm{I}_{\mathrm{Rm}}$ y $\mathrm{I}_{\mathrm{C}}$, definidos como el cociente entre los resultados obtenidos para la junta soldada y los obtenidos para el material base correspondiente. 
Tabla 7. Propiedades en tracción y embutido Olsen de las uniones soldadas por arco.

\begin{tabular}{|c|c|c|c|c|c|c|c|}
\hline Material & Proceso & $\begin{array}{c}\mathrm{R}_{\mathrm{m}} \\
{[\mathrm{MPa}]}\end{array}$ & $\mathrm{I}_{\mathrm{Rm}}$ & $\begin{array}{l}\text { Zona Rotura } \\
\text { Tracción }\end{array}$ & $\begin{array}{c}\mathrm{h}_{0} \\
{[\mathrm{~mm}]}\end{array}$ & $\mathrm{I}_{\mathrm{C}}$ & $\begin{array}{c}\text { Zona Rotura } \\
\text { Embutido }\end{array}$ \\
\hline $\mathrm{C}-\mathrm{Mn}$ & \multirow{4}{*}{ GMAW } & 435 & 0,77 & MBR & 7,1 & 0,95 & ZAC \\
\hline $\mathrm{Nb}$ & & 575 & 0,77 & MBR/ ZSC & 6,4 & 0,62 & $\mathrm{ZAC}$ \\
\hline $\mathrm{Ti}-\mathrm{Nb}$ & & 670 & 0,79 & MBR/ ZSC & 7,5 & 0,77 & $\mathrm{ZAC}$ \\
\hline Ti-Nb- T & & 510 & 0,70 & MBR/ ZSC & 7,9 & 0,71 & ZAC \\
\hline C-Mn & \multirow{4}{*}{ PAW } & 431 & 0,76 & MBR/ ZSC & 6,8 & 0,91 & $\mathrm{ZAC}$ \\
\hline $\mathrm{Nb}$ & & 694 & 0,91 & MBR/ ZSC & 6,6 & 0,65 & $\mathrm{ZAC}$ \\
\hline $\mathrm{Ti}-\mathrm{Nb}$ & & 612 & 0,72 & MBR/ ZSC & 5,9 & 0,61 & ZAC \\
\hline Ti-Nb- T & & 513 & 0,70 & MBR/ ZSC & 6,2 & 0,55 & $\mathrm{ZAC}$ \\
\hline
\end{tabular}

El índice $\mathrm{I}_{\mathrm{Rm}}$ no es otra cosa que la eficiencia de la junta [17]. Para aceros DP, dicha eficiencia en general es menor que 1, debido principalmente a la degradación de las propiedades en la ZAC. En general se acepta que una eficiencia de 0,8 es satisfactoria [18]. La resistencia mecánica se encontró entre 0,70 y 0,91 del valor del material base, siendo mejores los resultados para las chapas finas $(0,77-0,91)$, asociado al menor aporte térmico utilizado para su soldadura. Las fracturas se produjeron principalmente en la transición entre las ZSC/MBR, donde se observó la menor dureza y una estructura de ferrita y carburos. Estos resultados son consistentes con la disminución de la dureza en dicha zona, respecto de la original.

Para el caso del acero C-Mn la fractura se produjo en el MBR dado que la dureza en ambas zonas es similar. Las eficiencias de junta $\left(I_{R m}\right)$ obtenidas en este trabajo son similares, aunque levemente inferiores, a las obtenidas por Kaptustka [6] para un DP780 soldado en condiciones similares mediante GMAW. El acero DP empleado en dicho trabajo presenta Mn-Cr-Mo como elementos de aleación principales. Este autor observó también la presencia de zonas de ablandamiento en la ZAC. La diferencia en la eficiencia de las juntas reportada podría estar asociada a la presencia de Cr y Mo que aumentarían la resistencia al revenido [19], aumentando el costo del acero base utilizado.

En relación a la conformabilidad, se encontró entre un 0,55 y 0,95 del material base. El material más afectado fue el Ti-Nb-T soldado con PAW, asociado a una soldadura con alto calor aportado $\left(0,35 \mathrm{~kJ} . \mathrm{mm}^{-1}\right)$, mientras que el menos afectado fue el acero C-Mn. En todos los casos la fractura se produjo en forma paralela al cordón de soldadura, en la ZAC. Esto es otro indicio que la conformabilidad de soldaduras por fusión de aceros DP está controlada por el ablandamiento de la ZAC [20]. El acero al C-Mn fue el menos afectado, debido a que el efecto de endurecimiento por la fracción de martensita formada en el DP fue menor y por lo tanto la pérdida de ese efecto por desaparición de dicha fase.

Bajo las condiciones llevadas a cabo en este trabajo, se obtuvieron resultados satisfactorios para las uniones soldadas, siendo similares los resultados obtenidos para ambos procesos GMAW y PAW. El ablandamiento de ZAC es un factor determinante de las propiedades de las soldaduras por arco de estos materiales.

\subsection{Análisis de las soldaduras por resistencia}

En todos los casos la soldabilidad fue muy buena. No se presentaron imperfecciones visibles sobre la soldadura, ni excesivas expulsiones de material, presentando una apariencia superficial de los puntos muy buena. En la Figura 6, se presentan los perfiles de microdureza obtenidos sobre los puntos soldados con el proceso RSW. Las flechas quebradas indican los límites de ZF, ZAC y MB para cada material.

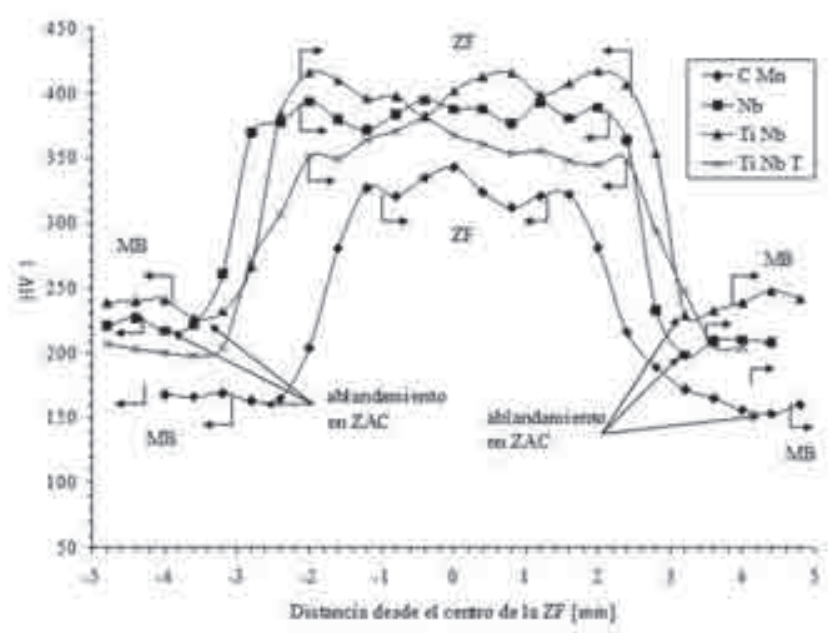

Figura 6. Perfil de microdureza de las uniones soldadas con RSW.

El perfil de microdureza tiene el aspecto típico para la soldadura RSW de aceros DP [21, 22, 23]. Los máximos registros de microdureza se encontraron en la zona de fusión (ZF). A su vez, las máximas durezas corresponden a las muestras $\mathrm{Nb}$ y Ti$\mathrm{Nb}$, lo cual coincide con los mayores valores de CEn y Pcm (ver Tabla 4) para estos aceros. En forma similar a las soldaduras por arco, los mínimos valores de microdureza se midieron en la 

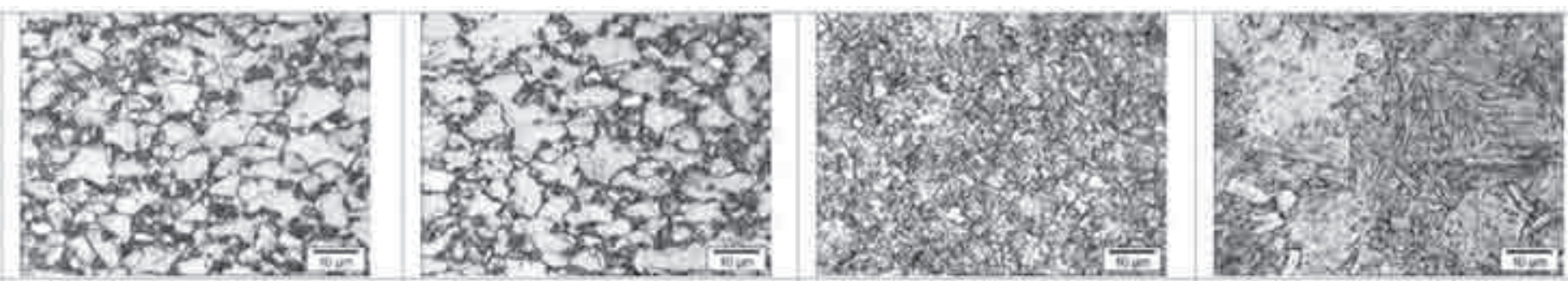

$\operatorname{MBR}(242 \pm 3 \mathrm{HV})$

ZSC ( $230 \pm 3 \mathrm{HV})$

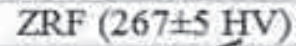

ZRG $(369 \pm 21 \mathrm{HV})$

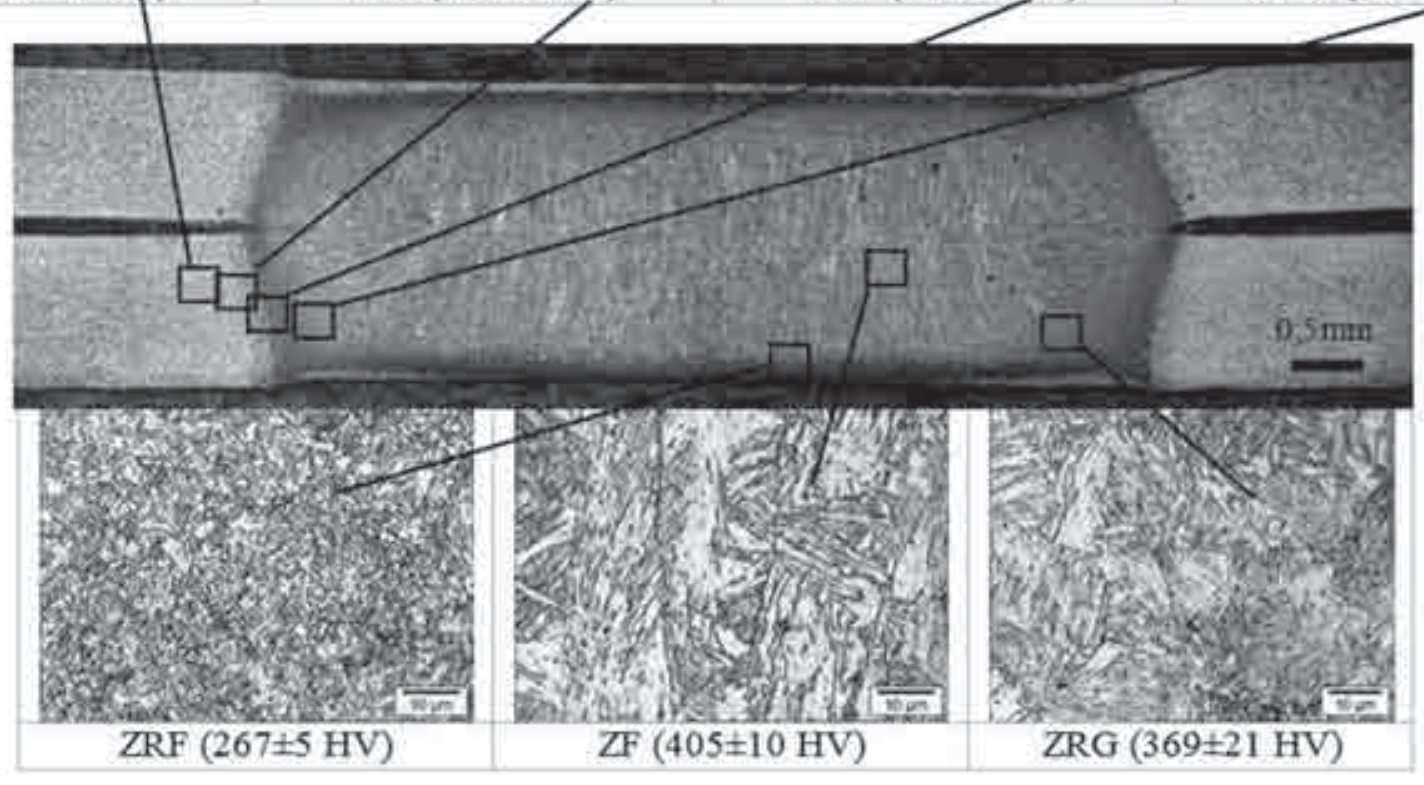

Figura 7. Microestructuras del punto soldado de la chapa Ti-Nb y microdureza promedio de cada zona.

ZAC, más precisamente en la zona cercana al MB.

En la Figura 7 se observan las microestructuras observadas en los puntos soldados por resistencia de la chapa identificada como Ti-Nb, y los valores de microdureza promedio de cada zona con su dispersión. Esta figura es representativa del resto de las soldaduras por resistencia, a excepción de las soldaduras C-Mn donde se observaron microestructuras de mayor tamaño, al igual que ocurriera con las soldaduras por arco. En las micrografías se distingue la zona ZF compuesta por granos columnares y estructuras martensíticas, debido a las altas velocidades de enfriamiento impuestas por los electrodos de cobre refrigerados [24].

Rodeando la ZF, se observa una ZRG y adyacente a ésta sigue una ZRF, que tiene asociada un afino de grano respecto del tamaño del material base. La microestructura en ésta zona es fundamentalmente ferrítica con escasa martensita y un tamaño de grano mucho más fino que el grano del metal base inicial. Luego, se encuentra una zona ZSC, donde la máxima temperatura fue levemente menor a $\mathrm{Ac}_{1}$ y se observa una estructura ferrítica de tamaño de grano similar al del metal base y martensita, a diferencia de los observado en las juntas soldadas por arco. Esta diferencia microestructural en la ZSC se podría asociar a la mayor velocidad de enfriamiento alcanzada en las soldaduras RSW, lo que no permitiría el revenido total de la martensita por falta de difusión. Del mismo modo que en las soldaduras GMAW y PAW, se distinguen zonas revenidas, MBR, en donde la martensita original de los aceros DP sufre una descomposición parcial por efecto de la temperatura, produciéndose una pérdida de dureza aparente, que luego se recupera hacia el metal base sin revenir.

En la Figura 6 se observó un leve ablandamiento en la ZAC cercana al MB de los puntos RSW. La diferencia entre la microestructura de la ZAC en las soldaduras por arco y las soldaduras por resistencia es que en la soldadura RSW no se pudieron apreciar zonas con características de las ZSC encontradas en las soldaduras GMAW y PAW. Indudablemente este comportamiento se debe a que las condiciones de enfriamiento en el proceso RSW son mucho más severas que las soldaduras por arco. Otro fenómeno consecuencia de las altas velocidades de enfriamiento del proceso RSW es la diferencia en la máxima dureza de las zonas fundidas entre las soldaduras RSW y PAW. Ambas fueron autógenas, es decir sin metal de aporte, pero para cada material estudiado la dureza de las soldaduras RSW son casi el doble de las soldaduras PAW.

De las pruebas de arrancamiento, peel tests, se pudo observar que las diferentes roturas se produjeron dentro de la ZAC. La ausencia de rotura en ZF conjuntamente con los modos de rotura que presentaron todos los aceros, indica una muy buena calidad de las soldaduras [25]. Como indica la Tabla 8, para todos los casos el tamaño de la zona fundida satisfizo los requerimientos de AWS D8.1 [25]. No se observó influencia del tipo de material sobre el valor obtenido. Asimismo se evaluó la indentación, tanto superior como inferior, medida de acuerdo a la mencionada especificación [24], siendo los resultados obtenidos 
Tabla 8. Tamaño de la zona de fusión e indentación para las soldaduras RSW.

\begin{tabular}{|l|c|c|c|c|c|}
\hline & $\begin{array}{c}\text { Requerimiento AWS } \\
\text { D8.1 } \\
{[\mathrm{mm}]}\end{array}$ & $\begin{array}{c}\text { Tamaño de zona } \\
\text { fundida } \\
{[\mathrm{mm}]}\end{array}$ & $\begin{array}{c}\text { Indentación } \\
\text { superior } \\
(\%)\end{array}$ & $\begin{array}{c}\text { Indentación } \\
\text { inferior } \\
(\%)\end{array}$ & $\begin{array}{c}\text { Requerimiento } \\
\text { AWS D8.1 } \\
(\%)\end{array}$ \\
\cline { 1 - 4 } $\mathrm{C}-\mathrm{Mn}$ & \multirow{3}{*}{$\mathrm{Nb}$} & 5,5 & 17 & 11 & $<30$ \\
\cline { 1 - 4 } & & 5,5 & 7 & 10 & $<30$ \\
\cline { 3 - 6 } $\mathrm{Ti}-\mathrm{Nb}$ & $\geq 5,0$ & 5,5 & 12 & 11 & $<30$ \\
\hline Ti-Nb-T & & 5,3 & 10 & 10 & $<30$ \\
\hline
\end{tabular}

sensiblemente menores que los máximos admisibles (Tabla 8). El comportamiento de los distintos materiales fue similar tanto respecto a la resistencia como al espesor. Solo el material C-Mn presentó un mayor valor, lo que podría estar asociado a su menor resistencia mecánica.

Por último en la Tabla 9 se compara el grado de ablandamiento, $\mathrm{DHV}_{\text {min }}(\%)$, para las juntas soldadas por arco y por resistencia. El grado de ablandamiento se define de acuerdo a la Ecuación (1),

$$
\Delta \mathrm{HV}_{\text {mit }}=\frac{\left(\mathrm{HV}_{\mathrm{MB}}-\mathrm{HV}_{\min }\right)}{\mathrm{HV}_{\mathrm{MB}}} .100
$$

Donde, $\mathrm{HV}_{\mathrm{MB}}$ es la dureza promedio del metal base y $\mathrm{HV}_{\text {min }}$ es la dureza mínima registrada en la unión soldada. El grado de ablandamiento es un índice que indica el la perdida de dureza debido a la degradación de la microestructura asociada al ciclo térmico de la soldadura. Se encuentra fuertemente relacionado con la eficiencia de la junta $\left(\mathrm{I}_{\mathrm{RM}}\right)$. Cuanto mayor es el valor de $\mathrm{DHV}_{\text {min }}$ mayor es el impacto de la soldadura en la resistencia del componente soldado. Índices similares se han empleado previamente [18]. En todos los casos $\mathrm{HV}_{\text {min }}$ se encontró en la ZAC de cada unión, más precisamente dentro de la zona subcrítica (ZSC).

Tabla 9. Grado de ablandamiento, $\mathrm{DHV}_{\text {min }}(\%)$, para cada junta.

\begin{tabular}{|l|c|c|c|}
\hline \multicolumn{4}{|c|}{ DHV $_{\text {min }}(\%)$} \\
\hline & GMAW & PAW & RSW \\
\hline C-Mn & 25 & 31 & 4 \\
\hline Nb & 30 & 28 & 8 \\
\hline Ti-Nb & 28 & 27 & 9 \\
\hline Ti-Nb-T & 37 & 37 & 5 \\
\hline
\end{tabular}

Estos resultados muestran el menor nivel de ablandamiento de la ZAC de las soldaduras RSW respecto de las soldaduras por arco. Esto estaría asociado a las altas velocidades de enfriamiento relacionadas con el proceso RSW. Asimismo, se observa que las muestras de mayor espesor ( $\mathrm{Ti}-\mathrm{Nb}-\mathrm{T}$ ) para las que se utilizó un mayor aporte térmico, mostraron un mayor grado de ablandamiento. En general puede decirse que el aumento en la velocidad de enfriamiento producirá un menor grado de ablandamiento y por lo tanto tendrá un efecto favorable sobre la eficiencia de la junta. En este sentido procesos y/o procedimientos de soldadura que favorezcan dicha situación tenderán a presentar resultados más satisfactorios. A su vez, el uso de materiales más resistentes al ablandamiento (revenido) también producirá un menor grado de ablandamiento.

Cabe destacar que si bien los fenómenos metalúrgicos observados para los diferentes materiales, tipos de junta y procesos analizados en el presente trabajo se encuentran asociados al ciclo térmico de la soldadura, y son por lo tanto esperables en otros procesos o condiciones de soldadura, la magnitud de los mismos y los efectos que presenten sobre las características y propiedades de las uniones soldadas variarán en general con las condiciones y los procesos de soldadura que se empleen.

\section{Conclusiones}

En el presente trabajo se mostró que durante la soldadura mediante PAW y GMAW de aceros DP de alta resistencia en chapa fina presenta buena soldabilidad. Los tamaños de las juntas soldadas por PAW fueron menores que los obtenidos para GMAW, debido a que el primer proceso es autógeno.

Asimismo, se producen variaciones microestructurales, vinculadas al ciclo térmico de la misma, que afectan las propiedades mecánicas del material base. Estos cambios producen una disminución de resistencia mecánica y conformabilidad. El índice $\mathrm{I}_{\mathrm{Rm}}$ (eficiencia de la junta), se encuentra entre un 0,70 y 0,80 para el proceso GMAW y 0,70 y 0,91 para el proceso PAW. La conformabilidad relativa a la del metal base $\left(\mathrm{I}_{\mathrm{C}}\right)$ fue de 0,60 a 0,95 para las soldaduras GMAW y 0,55 a 0,91 para las soldaduras PAW, dependiendo del material y del espesor, entre otros factores. La disminución de las propiedades del metal base de los aceros DP en la soldadura por arco que las zonas que son calentadas hasta temperaturas levemente inferiores a $\mathrm{A}_{\mathrm{C} 1}$ (ZSC) pierden la fracción de martensita propia del DP por revenido, con un leve aumento del tamaño de grano original, formándose una estructura de ferrita y carburos de baja dureza.

En cuanto a las soldaduras por resistencia de los distintos aceros DP estudiados se observó que tienen una soldabilidad excelente. Si bien se observa también una disminución de la dureza en la zona ZSC. La misma es sensiblemente inferior que lo observado en las soldaduras por arco. Esto estaría vinculado a la alta velocidad de enfriamiento alcanzada durante las soldaduras RSW.

Los ciclos térmicos generados y las características del material base de los aceros DP, definen la evolución microestructural en la zona afectada por el calor de las soldaduras de estos de aceros, lo que controla las propiedades finales de la unión soldada. 


\section{Agradecimientos}

Los autores desean agradecer a Ternium-Siderar y Brasmetal por la provisión de los aceros base analizados, a Conarco-ESAB y Air Liquide por la provisión de los consumibles de soldadura y a la compañía Welding Argentina por la asistencia en la soldadura de punto por resistencia. Así también a INTI-Mecánica y al personal del LAME por el apoyo para la realización del presente trabajo.

\section{Referencias}

[1] International Iron and Steel Institute, Committee of Automotive Applications, Advanced High Strenght Steels (AHSS): Application Guidelines. Versión 3, Bruselas, IISI, p. 2-1 - 3-27, 2006.

[2] Dilthey, U.; Ste, L. Multimaterial car body design: challenge for welding and joining, Science and Technology of Welding and Joining, U.K., v. 11, n. 2, p. 135-142, Sept. 2005.

[3] Delince, M.; Brechet, Y.; Embury, J.D.; Geers, M.G.D.; Jacques, P.J.; Pardo, T. Structure-property optimization of ultrafine-grained dual-phase steels using a microstructure-based strain hardening model, Acta Materialia, USA, v. 55, p. 23372350, Feb. 2007.

[4] Kumar, A.; Singh, S.; Ray, K. Influence of bainite/martensitecontent on the tensile properties of low carbon dual-phase steels, Materials Science and Engineering: A, USA, v. 474, n. 1-2, p. 270-282, Feb. 2008.

[5] Reis, R. P. and Scotti, A.; Fundamentos e prática de soldagem a plasma. Sao Paulo: Artliber, 2007.

[6] Kapustka, N.; Conrardy, C.; Babu, S.; Albright, C. Effect of GMAW Process and Material Conditions on DP 780 and TRIP 780 Welds, Welding Journal, USA, v. 87, n. 6, p. 135s-148s, Jun. 2008.

[7] Feng, Z.; Chiang, J.; Jiang, C.; Kuo M.; Sang, J. Weldability and Performance of GMAW Joints of Advanced High-Strength Steels (AHSS), Great Designs in Steel Seminar, www.autosteel. com.

[8] Lorusso, H.; Burgueño, A.; Svoboda, H. Propiedades Mecánicas y Caracterización Microestructural de diferentes Aceros Dual-Phase. In: SAM/CONAMET, $8^{\circ}, 2008$, Santiago de Chile. Proceedings Chile: 2008. p. 282-285.

[9] American Society for Testing and Materials. ASTM E 562: Standard Test Method for Determining Volume Fraction by Systematic Manual Point Count. Pennsylvania, 2008.

[10] American Society for Testing and Materials. ASTM E 384: Standard Test Method for Knoop and Vickers Hardness of Materials. Pennsylvania, 2010

[11] American Society for Testing and Materials. ASTM E 8M: Standard Test Method for Tension Testing of Metallic Materials [metric], Pennsylvania, 2004.

[12] American Society for Testing and Materials. ASTM E 643: Standard Test Method for Ball Punch Deformation of Metallic Sheet Materials. Pennsylvania, 2000.

[13] American Welding Society. AWS/SAE D8.9M: Recommended practices for test methods for evaluating the resitance spot welding behaviour of automotive sheet steel materials. Miami, 2002.
[14] International Standarization Organization. ISO 14270: Specimen dimensions and procedure for mechanized peel testing resistance spot, seam and embossed projection welds. Geneva, 2000.

[15] Yurioka, N., Suzuki, H., Ohshita, S., and Saito, S. Determination of necessary preheating temperature in steel welding. Welding Journal, USA, v. 62, n. 6, p. 147s-153s, Jun. 1983.

[16] Kou, S. Welding Metallurgy. 2. Ed. New Jersey: John Wiley \& Sons, 2003. p. 393-404.

[17] American Welding Society. AWS A3.0-94: Standard Welding Terms and Definitions. Miami, 1994.

[18] So, W.; Kang, M; Kim, D. Weldability of pelsed GMAW joints of $780 \mathrm{MPa}$ dual-phase steel, Archives of Materials Science and Engineering, Poland, v. 41, n. 1, p. 53-60, Jan. 2010. [19] Linnert, G. Welding Metallurgy, Carbon and Alloy Steels, Volume 1, Fundamentals. 4. Ed. Miami: American Welding Society, 1994. p. 345-347.

[20] Xia, M.; Kuntz, M.; Tian, Z.; Zhou, Y. Failure analysis on laser welds of dual phase steel in formability testing, Science and technology of welding and joining, London, v. 13, n. 4, p. 378-387, Apr. 2008.

[21] Dancette, S.; Fabregue, D.; Merlin, J.; Massardier-Jourdan, V.; Bouzekri, M. Welding of advanced high strength steels: study of the microstructures obtained and damage mechanism during cross tension test. In: New Developments on Metallurgy and Applications of High Strength Steels, 2008, Buenos Aires. Proceeding Buenos Aires: 2008: paper number 058.

[22] Ma, C.; Chena, D.L.; Bhole, S.D.; Boudreau, G.; Lee, A.; Biro, E. Microstructure and fracture characteristics of spotwelded DP600 steel, Materials Science and Engineering: A, USA, v. 485, n. 1-2, p. 334-346, Jun. 2008.

[23] Marya, M.; Gayden, X. Development of requirement for Resistance Spot Welding Dual-Phase (DP600) Steels Part 1-The cause of interfacial fracture, Welding Journal, USA, v. 84, n. 11, p. 172s-185s, Nov. 2005.

[24] Marashi P.; Pouranvari, M.; Sanaee, S.M.H.; Abedi, A.; Abootalebi, S.H.; Goodarzi, M. Relationship between failure behaviour and weld fusion zone attributes of austenitic stainless steel resistance spot welds, Materials Science and Technology, London, v. 24, n. 12, p. 1506-1512, Dec. 2008.

[25] American Welding Society. AWS/SAE D8.1M: Specification for automotive weld quality - resistance spot welding of steel. Miami, 2007. 